\title{
Didier Carnet, Jean-Pierre Charpy, Anglais des spécialités médicales ( $\left.L^{\prime}\right)$
}

Paris : Ellipses

François Maniez

\section{OpenEdition \\ Journals}

Édition électronique

URL : http://journals.openedition.org/asp/4772

DOI : 10.4000/asp.4772

ISSN : 2108-6354

\section{Éditeur}

Groupe d'étude et de recherche en anglais de spécialité

\section{Édition imprimée}

Date de publication : 9 mars 2016

Pagination : 169-172

ISSN : 1246-8185

\section{Référence électronique}

François Maniez, « Didier Carnet, Jean-Pierre Charpy, Anglais des spécialités médicales (L') », ASp [En ligne], 69 | 2016, mis en ligne le 01 mars 2016, consulté le 03 novembre 2020. URL : http:// journals.openedition.org/asp/4772 ; DOI : https://doi.org/10.4000/asp.4772

Ce document a été généré automatiquement le 3 novembre 2020.

Tous droits réservés 


\title{
Didier Carnet, Jean-Pierre Charpy, Anglais des spécialités médicales (L')
}

\author{
Paris : Ellipses
}

François Maniez

\section{RÉFÉRENCE}

Carnet, Didier \& Jean-Pierre Charpy. 2015. L'anglais des spécialités médicales. Paris :

Ellipses. 288 pages. ISBN 978-2-3400-0798-7. 
$1 \quad$ L'anglais des spécialités médicales arrive sur le marché doté de solides références, à commencer par la connaissance intime de l'anglais médical qu'ont ses auteurs, Didier Carnet et Jean-Pierre Charpy, qui publient tous deux dans ce domaine depuis de longues années. Il s'agit en effet de leur sixième opus commun aux éditions Ellipses en quinze ans, même si l'ouvrage est leur première publication en duo. Il faut également mentionner le gage de qualité que constitue la collaboration d'experts dans chacune des spécialités médicales traitées dans l'ouvrage, experts qui ont eux-mêmes pour la plupart validé des diplômes universitaires d'anglais et collaborent régulièrement avec les auteurs dans le cadre d'un dispositif pédagogique probablement unique sur

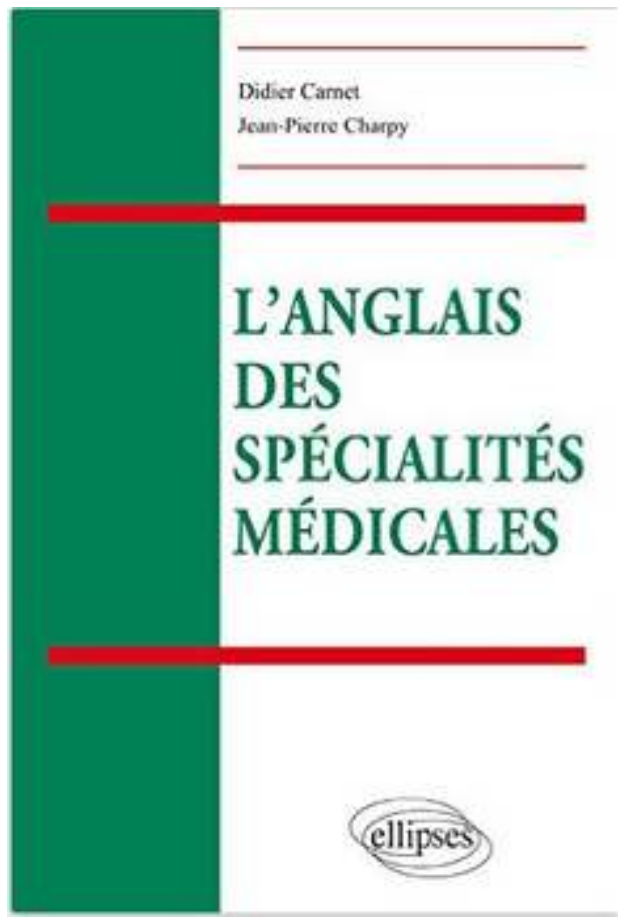
notre territoire. Ce dispositif, qui permet aux étudiants de la Faculté de Médecine de bénéficier de cours d'anglais associant médecins et enseignants de langue, a été mis en place par les auteurs à l'Université de Bourgogne.

2 Le but de l'ouvrage est de couvrir l'immense champ des spécialités médicales afin de permettre aux externes, aux internes et aux professionnels de santé en général de se familiariser avec les subtilités de la langue médicale spécialisée. Il a été conçu afin de leur faciliter l'acquisition des « compétences nécessaires dans une situation spécialisée pour répondre à des besoins lexicaux et communicationnels immédiats ».

3 Une caractéristique qui le distingue des précédents manuels publiés par ses auteurs est sa division en chapitres traitant de chacune des vingt-cinq spécialités choisies: anatomopathologie, cardiologie, chirurgie et anesthésie, dermatologie, endocrinologie, gastroentérologie, gériatrie, gynécologie et obstétrique, hématologie, imagerie médicale, maladies infectieuses, médecine générale, médecine interne et immunologie, médecine d'urgence, neurologie, oncologie, ophtalmologie, otorhinolaryngologie, pédiatrie, pneumologie, psychiatrie, rééducation, rhumatologie, santé publique, urologie et néphrologie. Chaque chapitre consacré à une spécialité comporte cinq rubriques, dans lesquelles les termes ou expressions de l'anglais sont systématiquement accompagnés de leur traduction française :

- un lexique de l'anatomie propre à la spécialité médicale abordée, présenté sous forme de glossaire ;

- un court lexique spécialisé de la physiologie permettant de cerner les termes utiles à la spécialité ;

- les mots-clés de la spécialité, généralement classés en plusieurs sous-rubriques (généralités, signes et symptômes, maladies, tests et examens, traitements...) ;

- les principaux sigles et acronymes ;

- les expressions les plus fréquemment utilisées lors de la consultation propre à chaque spécialité médicale (interrogatoire du patient et examen clinique). 

orthographiques entre l'anglais britannique et l'anglais américain. Dans de très nombreux cas, ils attirent également l'attention du lecteur sur la distinction entre le terme médical spécialisé qui est le plus souvent d'origine gréco-latine et le terme de vulgarisation utilisé par les médecins avec leurs patients, généralement formé à partir de racines germaniques (il s'agit par exemple de l'opposition entre trachea et windpipe).

5 L'atout principal de l'ouvrage est sans doute son exhaustivité, qui résulte à la fois de la longue expérience de ses auteurs de l'enseignement de l'anglais médical et de la révision attentive de chaque chapitre par un médecin expert de la spécialité concernée. La quantité d'information fournie est particulièrement impressionnante dans la rubrique consacrée aux sigles et aux acronymes, bon nombre d'entre eux étant absent des bases de données terminologiques consultables en ligne comme Termium ou le Grand Dictionnaire Terminologique. Dans un domaine où l'homonymie est notoire, la division en spécialités s'avère particulièrement utile dans la mesure où elle permet de dissocier des sigles identiques en fonction de la spécialité concernée ; par exemple, EC peut renvoyer à emergency contraception (dans le chapitre consacré à la gynécologie) ou à endothelial cell (dans le chapitre consacré à l'immunologie). Le problème épineux de la traduction de ces sigles (ou de l'utilisation du sigle anglais en français, phénomène relativement fréquent) est également traité de façon très complète.

6 L'autre point fort de l'ouvrage est la dernière rubrique de chaque chapitre, qui contient les expressions les plus fréquemment utilisées lors de la consultation propre à chaque spécialité médicale (interrogatoire du patient et examen clinique). Cette rubrique devrait s'avérer particulièrement utile aux médecins pratiquant en milieu anglophone, d'autant plus que le vocabulaire de la langue générale (qui est le plus susceptible d'être moins connu du médecin spécialiste) est abondamment représenté : la rubrique Physical examination du chapitre General medicine dresse par exemple la liste des vêtements susceptibles d'être portés par les patients et que le praticien peut être amené à lui demander d'ôter. Dans le même chapitre, la rubrique History taking comprend une sousrubrique intitulée Pain qui dresse un inventaire très complet de toutes les manières dont les patients peuvent décrire la douleur ressentie (burning/boring/gnawing/nagging/ stabbing/splitting/darting/ searing/stinging/tingling/gripping/squeezing/throbbing). Les appellations des différentes pathologies sont souvent décrites de manière très détaillée, donnant des informations terminologiques d'un tel degré de spécialisation qu'elles peuvent là aussi être absentes des bases de données de référence consultables en ligne (par exemple pour des termes tels que heart failure with [reduced/preserved] ejection fraction).

7 Ce qui fait la force de l'ouvrage de Didier Carnet et Jean-Pierre Charpy est donc clairement la quantité d'information de nature lexicale fournie dans les chapitres correspondant à chacune des vingt-cinq spécialités abordées, qu'il s'agisse des mots ou des expressions du vocabulaire de la langue générale ou de la langue spécialisée. Ce foisonnement d'informations lexicales est néanmoins susceptible de créer quelques difficultés qu'il est nécessaire de mentionner pour un utilisateur non spécialiste de la médecine, et qui justifieraient peut-être certains ajouts lors d'une édition ultérieure afin de satisfaire un public encore plus large.

8 Le format même de l'ouvrage implique fatalement une certaine duplication de l'information lexicale, notamment dans le domaine de l'anatomie et de la physiologie. Dans celui de l'anatomie notamment, il est bien difficile de distinguer ce qui relèverait 
spécifiquement de l'une des spécialités plutôt que d'une autre, les divers organes pouvant être le siège de manifestations propres à des maladies relevant de nombreuses spécialités. L'inclusion en début d'ouvrage d'un lexique consacré à l'anatomie générale aurait permis d'éviter la duplication du vocabulaire d'un moindre degré de spécialisation (par exemple les sous-rubriques The body, The skeleton et The internal organs, reproduites presque à l'identique dans onze des vingt-cinq chapitres) ainsi que certaines variations relatives au classement des vocables concernés : ainsi, les mots knee et ankle sont cités dans l'un des chapitres à l'intérieur de la sous-rubrique The body, dans un autre dans la sous-rubrique The skeleton, et dans un troisième dans la sousrubrique The joints. Par ailleurs, des termes tels que cerebellum, salivary glands et tonsils, cités dans la sous-rubrique The internal organs de la rubrique Anatomy du chapitre General medicine sont absents de la même sous-rubrique dans le chapitre Geriatrics. L'information portant sur un même sujet peut également être disséminée dans diverses sous-rubriques portant le même nom: ainsi les qualificatifs blinding/shooting/ excruciating/intractable cités dans la sous-rubrique Pain de la rubrique Keywords du chapitre General medicine (p. 68) viennent compléter la liste déjà très fournie (cf. infra) des différentes manières de décrire la douleur qui sont consignées dans la rubrique History taking du même chapitre.

9 Un autre problème concerne l'accès à la véritable mine d'informations lexicales que constitue chacun des chapitres de cet imposant manuel. La préface signale qu'il ne s'agit « pas seulement d'un dictionnaire ou d'un glossaire, mais d'un outil de référence pour la communication professionnelle en anglais médical». Cependant, la forme est plus proche de celle d'un thésaurus du fait du regroupement des informations sous la forme de chapitres correspondant aux diverses spécialités, et de l'absence du recours systématique à l'ordre alphabétique (en dehors des rubriques consacrées aux sigles et aux acronymes). De fait, la présence d'un bref index notionnel dans lequel auraient pu figurer certaines des sous-rubriques des mots-clés (headaches, pregnancy) ou de la consultation (the medical imaging session) aurait grandement facilité l'accès à l'information lexicale pour le non-spécialiste. À défaut d'un index, une table des matières plus détaillée mentionnant les rubriques et les sous-rubriques de chaque chapitre aurait également permis un accès plus rapide au contenu lexical extrêmement riche de l'ouvrage.

En résumé, ce manuel d'un format novateur a le mérite de présenter un matériau lexical très dense de façon structurée et très équilibrée. En effet, son organisation en fonction des diverses spécialités de la médecine et la similitude structurelle de ses chapitres font qu'il supporte tout à fait d'être lu de façon linéaire, ce qui distingue clairement son utilisation de celle d'un dictionnaire ou d'un simple lexique. S'il n'échappe pas à un certain degré de répétition de l'information lexicale, il est en revanche très complet et l'abondance des sigles et des acronymes justifie à elle seule son utilité en tant qu'ouvrage de référence. Sous sa forme actuelle, il convient bien au public visé, celui des professionnels de la médecine. Une réédition prenant davantage en compte les problématiques d'encodage et de décodage de l'angliciste cherchant à s'approprier le vocabulaire de la médecine en ferait certainement une référence incontournable dans le domaine de l'anglais médical. 


\section{AUTEURS}

\section{FRANÇOIS MANIEZ}

Directeur du Centre de recherche en terminologie et traduction (EA 4162), Université Lyon 2, francois.maniez@univ-lyon2.fr 